\title{
Transthoracic Needle Biopsy: How to Maximize Diagnostic Accuracy and Minimize Complications
}

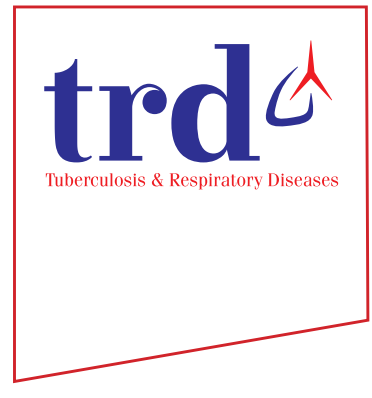

\author{
Chaeuk Chung, M.D., Ph.D. ${ }^{1}$ (D) , Yoonjoo Kim, M.D. ${ }^{2}$ and Dongil Park, M.D. ${ }^{2}$ (D) \\ ${ }^{1}$ Division of Pulmonary and Critical Care Medicine, Department of Internal Medicine, Chungnam National University, \\ Daejeon, ${ }^{2}$ Division of Pulmonary and Critical Care Medicine, Department of Internal Medicine, Chungnam National University \\ Hospital, Daejeon, Republic of Korea
}

Although transthoracic needle biopsy (TTNB) was introduced for lung biopsy about 40 years ago, it is still mainstay of pathologic diagnosis in lung cancer, because it is relatively inexpensive and can obtain tissue regardless of the tumor-bronchus relationship. With several technological advances, proceduralists can perform TTNB more safely and accurately. Utilizing ultrasound-guided biopsy for peripheral lesions in contact with the pleura and rapid onsite evaluation during the procedure are expected to make up the weakness of TTNB. However, due to the inherent limitations of the percutaneous approach, the incidence of complications such as pneumothorax or bleeding is inevitably higher than that of other lung biopsy techniques. Thorough understating of each biopsy modality and additional technique are fundamental for maximizing diagnostic accuracy and minimizing the complications.

Keywords: Image-Guided Transthoracic Needle Biopsy; Transthoracic Needle Biopsy; Lung Biopsy; Diagnostic Accuracy; Complication

\section{Introduction}

With the increase in lung cancer screening using lowdose computed tomography (CT), the detection of asymptomatic small pulmonary lesions has been increasing ${ }^{1,2}$. In addition, there is an increasing number of cases being conducted to assess driver mutations, resistance mechanisms,

Address for correspondence: Dongil Park, M.D.

Division of Pulmonary and Critical Care Medicine, Department of Internal Medicine, Chungnam National University Hospital, 282

Munhwa-ro, Jung-gu, Daejeon 35015, Republic of Korea

Phone: 82-42-280-6816, Fax: 82-42-222-7141

E-mail: rahm3s@gmail.com

Received: Dec. 3, 2020

Revised: Dec. 3, 2020

Accepted: Dec. 4, 2020

Published online: Dec. 4, 2020

(9) It is identical to the Creative Commons Attribution Non-Commercial License (http://creativecommons.org/licenses/by-nc/4.0/). and programmed death-ligand 1 expression in order to find the therapeutic options in case of disease progression during treatment ${ }^{3,4}$. National Comprehensive Cancer Network guideline recommends radial endobronchial ultrasound (EBUS), navigational bronchoscopy, and transthoracic needle biopsy (TTNB) as biopsy methods for peripheral pulmonary nodules ${ }^{5}$. In case of endobronchial approaches such as radial EBUS, serious complications are rare, however, there are limitations in which availability and diagnostic accuracy highly depend on tumor-bronchus relationship ${ }^{6-9}$. In imageguided TTNB, the incidence of pneumothorax, which is the most common complication, is 10 times higher than the endobronchial approaches such as radial EBUS and navigation bronchoscopy, and other complications such as hemorrhage are also higher. However, it is still one of the most commonly used and important biopsy techniques for peripheral pulmonary lesions because it is relatively inexpensive and has the advantage of obtaining a tissue regardless of tumor-bronchus relationship ${ }^{7}$. In this article, we will review each technique, and find out how to maximize the diagnostic accuracy and minimize complications. 


\section{How to Maximize Diagnostic Accuracy}

According to the guidance modality that leads the biopsy needle into the target lesion, the image-guided TTNB can be divided into conventional computed tomography-guided TTNB, computed tomography fluoroscopy-guided TTNB, and cone beam computed tomography-guided TTNB. In addition, it also can be divided into fine needle aspiration (FNA), core needle biopsy, coaxial needle technique, and single needle technique depending on the type of biopsy needle used for TTNB. To maximize the diagnostic accuracy, it is crucial to select an appropriate guidance modality and a biopsy needle considering the characteristics of the target lesion and the availability of resources in each institute and the operator. It can be helpful to use additional techniques such as rapid onsite evaluation (ROSE) to improve a diagnostic accuracy.

\section{Conventional computed tomography vs. Computed tomography fluoroscopy}

The first step to maximize diagnostic accuracy is selecting the most appropriate image guidance modality. Conventional computed tomography (CCT) is well-established TTNB guidance modality for the diagnosis of pulmonary lesions. It allows to determine the skin puncture site, needle insertion angle, and confirm the needle tip placement. It has been used since the 1980s, and recently published studies showed excellent diagnostic accuracy $(96.1 \%-96.7 \%)$ which is comparable to other latest modalities ${ }^{10-12}$. Even the results of CCT-guided TTNB for lesions smaller than $1 \mathrm{~cm}$ or ground glass nodule (GGN) showed more than $90 \%$ diagnostic accuracy ${ }^{13}$. However, there are technical difficulties in patients with large respiratory motion or inability to breathe coordinately due to lack of real-time capability. In particular, lesions located close to the diaphragm are technically challenging due to respiratory movement when using CCT, thereby the diagnostic accuracy decrease 5 . Furthermore, in case of small subpleural lesions, if a large pneumothorax occurs prior to biopsy, tissue acquisition may fail (Figure 1). To overcome these shortcomings, computed tomography fluoroscopy (CTF), which provides real-time CT images, was introduced in the 1990s. Since CTF can access the target lesion while checking the location of the biopsy needle in real time, it is possible to perform relatively accurate procedure even in difficult lesions with large respiratory motion or in patients with poor respiratory control. In prospective trials comparing CCT and CTF, though CTF showed significant reduction in procedure time and in the number of pleura punctures, it did not show improvement in diagnostic accuracy. In addition, there is a disadvantage that the operator is exposed to radiation during CTF-guided TTNB ${ }^{11}$.
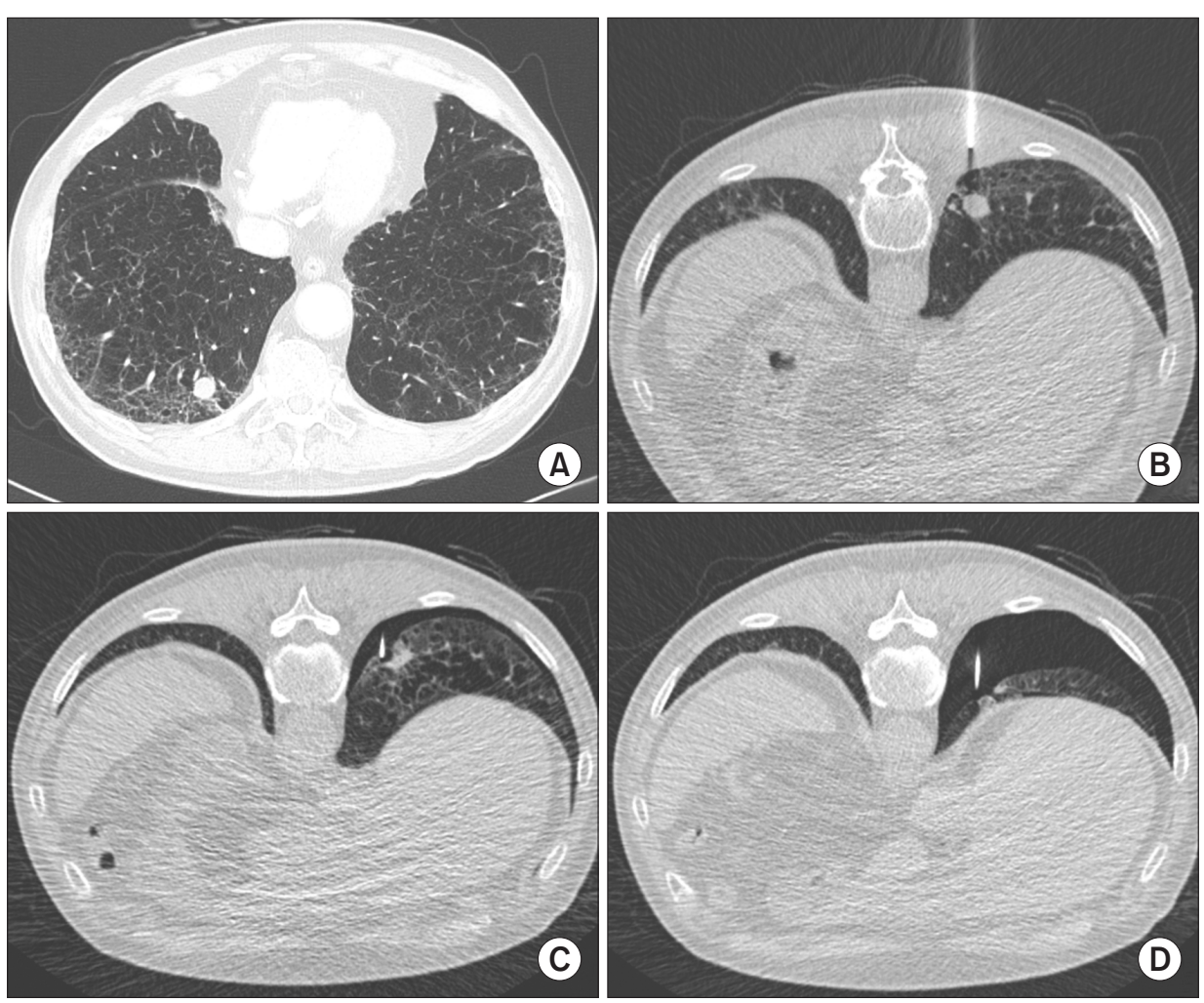

Figure 1. A technical failure in conventional computed tomography-guided transthoracic needle biopsy. (A) Small subpleural lesion with peripheral emphysema and fibrosis in right lower lobe. (B) A coaxial needle was inserted through the skin entry point in the prone position. (C, D) A pneumothorax occurred after a pleural puncture, and the location of the target lesion deviated as the size of the pneumothorax increased. 


\section{Computed tomography fluoroscopy vs. Cone beam computed tomography}

In the last decade, cone beam computed tomography (CBCT) which could provide fluoroscopy guidance and computed tomography images using a cone beam X-ray tube and a flat-panel detector was introduced. With this modality, the operator can confirm the exact location of target lesion and the path of the biopsy needle in real time. In addition, by using a dedicated software with color spot marking on preprocedural CBCT images, it allows to access a pure GGN or small nodules which are not visible on fluoroscopy. However, due to the low image quality of fluoroscopy, diagnostic accuracy possibly decrease when the target lesion is quite small or pure GGNs despite using the integrated virtual guidance image on fluoroscopic view (Bull's eye view) ${ }^{14}$. In a retrospective study including pulmonary lesions less than $1 \mathrm{~cm}(\mathrm{n}=172)$, the diagnostic accuracy of fluoroscopically invisible nodules was $76.7 \%$, which was significantly lower than $89.1 \%$ of visible nodules $^{15}$. In CTF, the operator could experience discomfort during the procedure due to the limited gantry, which is smaller than the C-arm gantry, and since the needle path and the location of the target lesion can be visualized only on the axial plane, the procedure probably becomes challenging if breathing cooperation is not sufficient. However, since real-time CT images are available, CTF possibly has an advantage over CBCT for small lesions or GGNs that are not directly visible on fluoroscopic view ${ }^{15,16}$. To the best of our knowledge, there has not been a prospective study comparing the diagnostic performance of CTF and CBCT which offer real-time capability. A retrospective study which compared CTF and CBCT showed that the diagnostic accuracy of CTF group and CBCT group were $94 \%$ and $96 \%$, respectively without significant difference in complication rate ${ }^{17}$. Well designed randomized trial is required to confirm which modality is superior for the small and GGN lesions especially adjacent to the pleura.

\section{Ultrasound vs. Computed tomography}

Ultrasound (US)-guided TTNB is another decent option for the peripheral lesions with pleural contact. Studies published by 2015, showed the excellent overall pooled diagnostic accuracy of $88.7 \%$ and sensitivity for malignancy of $91.5 \%$, respectively. Notably, the incidence of pneumothorax, the most common complication, was only $4.4 \%$, which is superior to other TTNB modalities at $20 \%^{13}$. The study with lung lesions contacting the pleura also demonstrated that the overall complication rate was lower in US-guided TTNB (7\%) than in CT-guided TTNB (24\%). Subgroup analysis showed USguided TTNB has fewer complications for lesions 31-50 mm $(\mathrm{p}=0.029)$, higher sample adequacy for lesions $10-30 \mathrm{~mm}$ $(\mathrm{p}=0.0032)$, and shorter procedural times for lesions 10-30 $\mathrm{mm}(\mathrm{p}<0.001)$ compared to CT-guided biopsy ${ }^{18}$. An additional benefit of US-guided biopsy is that complications such as pneumothorax can be identified immediately after procedure. The lung sliding on US, which means that visceral pleura and parietal pleura are not separated, can perfectly exclude the occurrence of pneumothorax $(100 \% \text { negative predictive value })^{19}$.

\section{Fine needle aspiration vs. Core needle biopsy}

FNA biopsy provides cytology specimens, while core needle biopsy provides tissues suitable for histological examination usually requiring biopsy needles with a larger diameter ${ }^{2,3}$. Core biopsy has shown slightly higher overall diagnostic accuracy (93\%), overall sensitivity (89\%), and specificity (97\%) comparing to the results with $\mathrm{FNA}^{20}$. However, there is no difference in diagnostic accuracy when ROSE of cytological samples is available in lung cancer lesions. If formalin-fixed paraffin-embedded tissue is used for molecular testing of lung cancer, core needle biopsy specimens are more likely to be adequate than FNA specimens ${ }^{3}$. In addition, core needle biopsy is preferred in suspected benign lesions which should be confirmed by a specific pathological finding such as necrotizing granulomas, or in malignant lesions that require confirmation of the cellular architecture for a definite diagnosis and subtype classification such as lymphoma ${ }^{21,22}$.

\section{Needle size}

There has not been a strong evidence that the largerdiameter biopsy needles improve diagnostic accuracy ${ }^{23}$. Nevertheless, for some lesions, the needle size possibly affects the diagnostic accuracy because in the case of necrotic lesions, it is common to obtain tissue from non-necrotic viable lesions. In our institution, 16 gauze or 18 gauze cutting biopsy needles are preferred rather than 20 gauze when necrosis is present, or when the cellular architectures are required for a definite diagnosis.

\section{Coaxial needle technique vs. Single needle technique}

Using the single needle technique, the operator introduces a biopsy needle though pleura repeatedly to obtain multiple specimens. Otherwise, the coaxial needle technique has advantage to allow multiple samplings though the outer needle sheath avoiding repeated punctures of the pleura and parenchymal structures after removing the inner stylet (Figure 2) ${ }^{24}$. However, the pleura and the parenchyma can be damaged due to increase of the diameter and the needle dwell time in which the pleura being penetrated by the axial needle ${ }^{25}$. In a meta-analysis, coaxial needles showed a tendency to increase complications, but there was no statistical significance. A recently published retrospective study showed that in patients with lesion size of $<1.5 \mathrm{~cm}$ and needle path length of $\geq 4 \mathrm{~cm}$, the coaxial technique achieved a higher diagnostic accuracy 


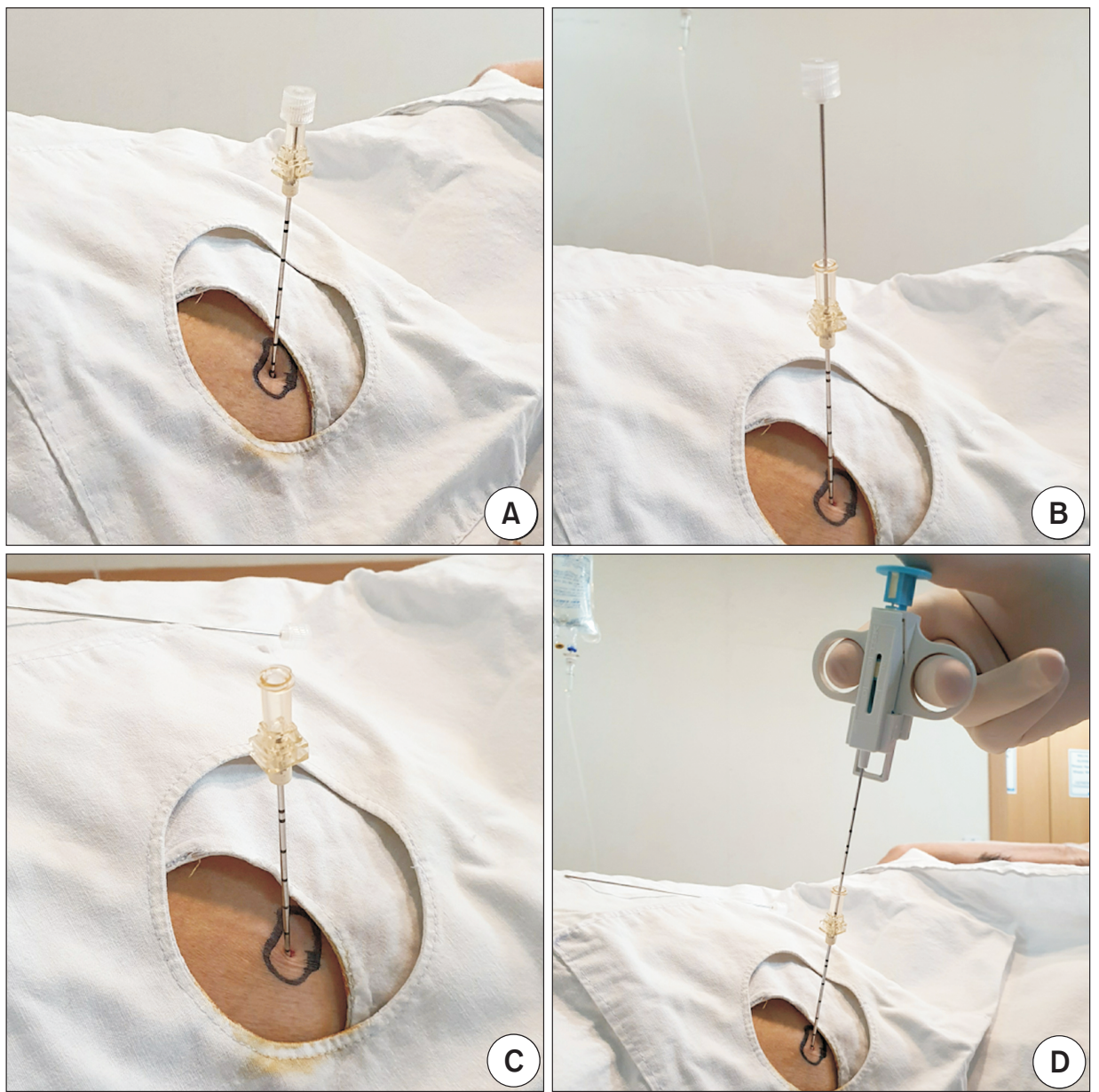

Figure 2. Coaxial needle technique. (A) An introducer needle was inserted into the intrathoracic target lesion. (B, C) The inner stylet was removed. (D) Then, a semi-automatic cutting needle (Stericut, TSK Laboratory, Tochigi, Japan) was inserted via the coaxial introducer, and multiple biopsies were performed.
(95.5\% vs. $72.7 \%, \mathrm{p}=0.023)$ and rather a lower pneumothorax incidence $^{26}$.

\section{Rapid on-site evaluation}

On-site cytology evaluation has shown to improve a diagnostic accuracy and reduce needle passes in image-guided transthoracic needle aspiration ${ }^{27,28}$. It is not clear whether additional diagnostic gain could be obtained when a core biopsy needle is used. However, in the case of FNA, the combination with ROSE provides a comparable diagnostic accuracy to that of a core needle biopsy for malignant lesions. Capalbo et al. ${ }^{29}$ even showed superior diagnostic accuracy compared to core needle biopsy in the presence of a pathologist for malignant lesions.

\section{Novel guidance modality: electromagnetic guidance TTNB}

In recent years, an electromagnetic navigation bronchoscopy (ENB) system that can guide transthoracic needle biopsy has become available. Electromagnetic guidance TTNB
(EM-guided TTNB) basically uses the same principle as ENB, however, it guides the biopsy needle into the target lesion instead of bronchoscopic biopsy tools ${ }^{30-32}$. In 2019, the result of a retrospective, multicenter study using EM-guided TTNB was published. This study consisted of seven academic and three community medical centers and the data from 95 participants were analyzed. The overall diagnostic yield was $73.7 \%$, and the diagnostic yield for malignancy was $68.8 \%$. However, in this study, 34 cases with non-definite pathologic results such as cellular atypia or lung parenchyma were excluded from the analysis which might have been counted as false negative cases. Therefore, the possibility that the diagnostic yield has been overestimated cannot be ruled out. The results should be confirmed by the prospective trial in order to determine the diagnostic performance of EM-guided $\mathrm{TTNB}^{30,33}$.

\section{How to Minimize Complications}

The major complications of TTNB and their respective incidence rates are as follows: pneumothorax (20\%), hemorrhage and hemoptysis (11\%), air embolism (0.02-1.8\%), tumor 
Table 1. Major complications of transthoracic needle biopsy

\begin{tabular}{|lc|}
\hline & Incidence (\%) \\
\hline Pneumothorax & 20 \\
Hemorrhage and hemoptysis & 11 \\
\hline Air embolism & $0.02-1.8$ \\
Tumor seeding & $0.12-0.061$ \\
\hline
\end{tabular}

seeding (0.12-0.061\%) (Table 1). To minimize complications, it is most important to avoid the procedure of patients with risk factors whenever possible. It is also important to select an appropriate guidance modality and use techniques to reduce complications. Here, we will review the two major complications (pneumothorax/hemorrhage), and how to minimize them.

\section{Pneumothorax}

Pneumothorax is the most common complication associated with TTNB procedure and the incidence rate is about $20 \%{ }^{13}$. Risk factors for pneumothorax, which have been proven by many studies, are as follows: presence of emphysema, percent emphysema, longer needle path length (depth of lesion), lower lobe location, core needle biopsy, repeated pleural puncture, small lesion $(\leq 2 \mathrm{~cm})$, lesion depth $(0.1$ to 2 $\mathrm{cm}$ ), old age $\mathrm{e}^{13,34-37}$. In order to minimize pneumothorax, it is also important to select an appropriate guidance modality. Since the lungs move according to breathing, selecting a realtime guidance modality such as CTF or CBCT can be helpful for reducing a pneumothorax especially in patients with poor respiratory holding capacity or with severe emphysema. In our experience, when compared with CCT, CTF possibly lower the incidence of pneumothorax by reducing the number of pleural punctures. Even in the event of pneumothorax, realtime capacity of CTF may decrease the technical failure ${ }^{11}$. As previously described, the incidence of pneumothorax of USguided TTNB has been reported only about $4 \%$, so it should be considered for lesions with pleural contact ${ }^{13,18}$. There are several techniques to minimize the incidence of pneumothorax. Rapid needle-out patient-rollover technique reduces the incidence of pneumothorax and chest catheter replacement by changing the patient's position into the puncture site down within ten seconds ${ }^{38,39}$. Sealing the biopsy needle track, using the autologous blood patch injection or hydrogel plug technique while removing the needle after obtaining the tissue, reduces the incidence of pneumothorax and chest catheter replacement ${ }^{40-42}$. In addition to these materials, track sealing with saline is also effective to reduce the incidence and chest tube insertion $^{43}$. In another study, saline sealing did not reduce the incidence, but significantly reduced pneumothorax size and chest tube replacement ${ }^{44}$.

\section{Delayed pneumothorax}

Delayed pneumothorax is defined as a pneumothorax detected three to four hours after the procedure, and the incidence rate has been reported up to $4.5 \%$. The mechanism is unclear, but it has been suggested that it may occur due to displacement of small blood clots on the penetrated pleura ${ }^{45}$. In some institutes, patients are discharged at the same day they receive the TTNB procedure due to high medical costs. Dennie et al. ${ }^{36}$ evaluated the safety of early discharge after TTNB $(\mathrm{n}=506)$. In this prospective study, patients were discharged after 30 minutes post-biopsy chest radiography if there was no pneumothorax. Delayed pneumothoraces were detected in seven patients $(1.4 \%)$. However, the actual incidence rate could have been higher than this because the discharged patient revisited hospitals only when they had symptoms ${ }^{36}$. Although there was no serious complication due to delayed pneumothorax in this study, delayed pneumothorax could be fatal and for this reason, in many hospitals, TTNB is still performed in the inpatient setting. Recently we performed a prospective interventional trial to predict a delayed pneumothorax using a lung ultrasound, and the results were promising (unpublished). We will publish the paper within the next few months.

\section{Pulmonary hemorrhage (hemoptysis)}

Pulmonary hemorrhage is the second common complication, and the incidence rate is about $11 \%{ }^{13}$. Risk factors, which have been proven by multiple studies, are as follows: core needle biopsy, small lesion $(\leq 2 \mathrm{~cm})$, lesion depth $(\geq 2.1$ or $>3 \mathrm{~cm})$, main pulmonary artery diameter/ascending aorta diameter ratio > 1, emphysema ${ }^{34-37,46}$. Recently, Kim et al. ${ }^{47}$ demonstrated that bronchovascular injuries are significantly associated with clinically significant hemoptysis after TTNB, which have been considered risk factors (but have not been scientifically proven). Multivariate analysis showed that the potential penetration of a pulmonary vessel $1 \mathrm{~mm}$ or larger at firing (odds ratio [OR], 3.874; 95\% confidence interval [CI], 1.072-13.997; $\mathrm{p}=0.039$ ), and cutting injury of a pulmonary vessel $1 \mathrm{~mm}$ or larger (OR, 6.920; 95\% CI, 1.728-27.711; p=0.006) or a bronchial structure (OR, 4.604; 95\% CI, 1.194-17.755; $\mathrm{p}=0.027$ ) are significant risk factors ${ }^{47}$. Therefore, the biopsy needle should be carefully inserted while avoiding the structures mentioned above. Also, recently, open bronchus sign was reported to be a risk factor for hemoptysis ${ }^{48}$. Intriguingly, the open bronchus sign, which is a similar concept to CT bronchus sign, is both a risk factor for hemoptysis and a favorable factor for diagnostic accuracy of radial EBUS and ENB ${ }^{8,9,48,49}$. Therefore, endobronchial approaches might be better choice for lesions with CT bronchus sign. 


\section{Conclusion}

In order to maximize diagnostic accuracy, it is crucial to select an appropriate guidance modality and instruments. In terms of complications, it is important to avoid risk factors whenever possible, and the additional techniques can be used together to reduce complications during the procedure.

\section{Authors' Contributions}

Conceptualization: Park D. Writing - original draft preparation: Park D. Writing - review and editing: Park D, Chung C, Kim Y. Approval of final manuscript: all authors.

\section{Conflicts of Interest}

No potential conflict of interest relevant to this article was reported.

\section{Funding}

This work was supported by Bio \& Medical Technology Development Program through the of the National Research Foundation (NRF) \& funded by the Korean government (NRF-2019M3E5D1A02068566).

\section{References}

1. Duffy SW, Field JK. Mortality reduction with low-dose CT screening for lung cancer. N Engl J Med 2020;382:572-3.

2. Kim YC, Won YJ. The development of the Korean Lung Cancer Registry (KALC-R). Tuberc Respir Dis 2019;82:91-3.

3. Schneider F, Smith MA, Lane MC, Pantanowitz L, Dacic S, Ohori NP. Adequacy of core needle biopsy specimens and fine-needle aspirates for molecular testing of lung adenocarcinomas. Am J Clin Pathol 2015;143:193-200.

4. Kage H, Kohsaka S, Shinozaki-Ushiku A, Hiraishi Y, Sato J, Nagayama K, et al. Small lung tumor biopsy samples are feasible for high quality targeted next generation sequencing. Cancer Sci 2019;110:2652-7.

5. NCCN guideline non-small cell lung cancer, 2021 (version 1) [Internet]. Plymouth Meeting: National Comprehensive Cancer Network; 2020 [cited 2020 Nov 10]. Available from: https://www.nccn.org/.

6. Katsis JM, Rickman OB, Maldonado F, Lentz RJ. Bronchoscopic biopsy of peripheral pulmonary lesions in 2020: a review of existing technologies. J Thorac Dis 2020;12:3253-62.

7. Deng CJ, Dai FQ, Qian K, Tan QY, Wang RW, Deng B, et al. Clinical updates of approaches for biopsy of pulmo- nary lesions based on systematic review. BMC Pulm Med 2018;18:146.

8. Minezawa T, Okamura T, Yatsuya H, Yamamoto N, Morikawa $\mathrm{S}$, Yamaguchi T, et al. Bronchus sign on thin-section computed tomography is a powerful predictive factor for successful transbronchial biopsy using endobronchial ultrasound with a guide sheath for small peripheral lung lesions: a retrospective observational study. BMC Med Imaging 2015;15:21.

9. Folch EE, Pritchett MA, Nead MA, Bowling MR, Murgu SD, Krimsky WS, et al. Electromagnetic navigation bronchoscopy for peripheral pulmonary lesions: one-year results of the prospective, multicenter NAVIGATE study. J Thorac Oncol 2019;14:445-58.

10. Loh SE, Wu DD, Venkatesh SK, Ong CK, Liu E, Seto KY, et al. CT-guided thoracic biopsy: evaluating diagnostic yield and complications. Ann Acad Med Singap 2013;42:285-90.

11. Kim GR, Hur J, Lee SM, Lee HJ, Hong YJ, Nam JE, et al. CT fluoroscopy-guided lung biopsy versus conventional CT-guided lung biopsy: a prospective controlled study to assess radiation doses and diagnostic performance. Eur Radiol 2011;21:232-9.

12. Jeon MC, Kim JO, Jung SS, Park HS, Lee JE, Moon JY, et al. CTguided percutaneous transthoracic needle biopsy using the additional laser guidance system by a pulmonologist with 2 years of experience in CT-guided percutaneous transthoracic needle biopsy. Tuberc Respir Dis 2018;81:330-8.

13. DiBardino DM, Yarmus LB, Semaan RW. Transthoracic needle biopsy of the lung. J Thorac Dis 2015;7:S304-16.

14. Fior D, Vacirca F, Leni D, Pagni F, Ippolito D, Riva L, et al. Virtual guidance of percutaneous transthoracic needle biopsy with C-arm cone-beam CT: diagnostic accuracy, risk factors and effective radiation dose. Cardiovasc Intervent Radiol 2019;42:712-9.

15. Hwang EJ, Kim H, Park CM, Yoon SH, Lim HJ, Goo JM. Cone beam computed tomography virtual navigation-guided transthoracic biopsy of small $(\leq 1 \mathrm{~cm})$ pulmonary nodules: impact of nodule visibility during real-time fluoroscopy. Br J Radiol 2018;91:20170805.

16. Lee SM, Park CM, Lee KH, Bahn YE, Kim JI, Goo JM. C-arm cone-beam CT-guided percutaneous transthoracic needle biopsy of lung nodules: clinical experience in 1108 patients. Radiology 2014;271:291-300.

17. Rotolo N, Floridi C, Imperatori A, Fontana F, Ierardi AM, Mangini M, et al. Comparison of cone-beam CT-guided and CT fluoroscopy-guided transthoracic needle biopsy of lung nodules. Eur Radiol 2016;26:381-9.

18. Lee MH, Lubner MG, Hinshaw JL, Pickhardt PJ. Ultrasound guidance versus CT guidance for peripheral lung biopsy: performance according to lesion size and pleural contact. AJR Am J Roentgenol 2018;210:W110-7.

19. Lichtenstein DA. Lung ultrasound (in the critically ill) superior to CT: the example of lung sliding. Korean J Crit Care Med 2017;32:1-8.

20. Anzidei M, Porfiri A, Andrani F, Di Martino M, Saba L, Cata- 
lano C, et al. Imaging-guided chest biopsies: techniques and clinical results. Insights Imaging 2017;8:419-28.

21. Cham M, Henschke CI, Yankelevitz DF. Biopsy procedures of the lung, mediastnum, and chest wall. In: Kandarpa K, Machan L, editors. Handbook of interventional radiologic procedures. 4th ed. Philadelphia: Lippincott Williams \& Wilkins; 2011. p. 478-85.

22. Sklair-Levy M, Polliack A, Shaham D, Applbaum YH, Gillis S, Ben-Yehuda D, et al. CT-guided core-needle biopsy in the diagnosis of mediastinal lymphoma. Eur Radiol 2000;10:714-8.

23. Moore W, Sawas A, Lee C, Ferretti J. Needle gauge and cytological yield in CT-guided lung biopsy. ISRN Pulmonol 2011;2011:970813.

24. Yankelevitz DF, Vazquez M, Henschke CI. Special techniques in transthoracic needle biopsy of pulmonary nodules. Radiol Clin North Am 2000;38:267-79.

25. Ko JP, Shepard JO, Drucker EA, Aquino SL, Sharma A, Sabloff $B$, et al. Factors influencing pneumothorax rate at lung biopsy: are dwell time and angle of pleural puncture contributing factors? Radiology 2001;218:491-6.

26. Zhang L, Shi L, Xiao Z, Qiu H, Peng P, Zhang M. Coaxial technique-promoted diagnostic accuracy of CT-guided percutaneous cutting needle biopsy for small and deep lung lesions. PLoS One 2018;13:e0192920.

27. Anila KR, Nayak N, Venugopal M, Jayasree K. Role of rapid onsite evaluation in CT-guided fine needle aspiration cytology of lung nodules. J Cytol 2018;35:229-32.

28. Marotti JD, Rao KP, Brister KJ, Gutmann EJ, Tsapakos MJ, Sheiman R, et al. Cytologic rapid on-site evaluation of transthoracic computed tomography-guided lung needle biopsies: who should perform ROSE? A cross-institutional analysis of procedural and diagnostic outcomes. J Am Soc Cytopathol 2015;4:160-9.

29. Capalbo E, Peli M, Lovisatti M, Cosentino M, Mariani P, Berti E, et al. Trans-thoracic biopsy of lung lesions: FNAB or CNB? Our experience and review of the literature. Radiol Med 2014;119:572-94.

30. Yarmus LB, Arias S, Feller-Kopman D, Semaan R, Wang KP, Frimpong B, et al. Electromagnetic navigation transthoracic needle aspiration for the diagnosis of pulmonary nodules: a safety and feasibility pilot study. J Thorac Dis 2016;8:186-94.

31. Arias S, Lee H, Semaan R, Frimpong B, Ortiz R, Feller-Kopman D, et al. Use of electromagnetic navigational transthoracic needle aspiration (E-TTNA) for sampling of lung nodules. J Vis Exp 2015:e52723.

32. Mallow C, Lee H, Oberg C, Thiboutot J, Akulian J, Burks AC, et al. Safety and diagnostic performance of pulmonologists performing electromagnetic guided percutaneous lung biopsy (SPiNperc). Respirology 2019;24:453-8.

33. Thiboutot J, Lee HJ, Silvestri GA, Chen A, Wahidi MM, Gilbert $\mathrm{CR}$, et al. Study design and rationale: a multicenter, prospective trial of electromagnetic bronchoscopic and electromagnetic transthoracic navigational approaches for the biopsy of peripheral pulmonary nodules (ALL IN ONE Trial). Contemp Clin Trials 2018;71:88-95.

34. Heerink WJ, de Bock GH, de Jonge GJ, Groen HJ, Vliegenthart $\mathrm{R}$, Oudkerk M. Complication rates of CT-guided transthoracic lung biopsy: meta-analysis. Eur Radiol 2017;27:138-48.

35. Chami HA, Faraj W, Yehia ZA, Badour SA, Sawan P, Rebeiz $\mathrm{K}$, et al. Predictors of pneumothorax after CT-guided transthoracic needle lung biopsy: the role of quantitative CT. Clin Radiol 2015;70:1382-7.

36. Dennie CJ, Matzinger FR, Marriner JR, Maziak DE. Transthoracic needle biopsy of the lung: results of early discharge in 506 outpatients. Radiology 2001;219:247-51.

37. Yeow KM, Su IH, Pan KT, Tsay PK, Lui KW, Cheung YC, et al. Risk factors of pneumothorax and bleeding: multivariate analysis of 660 CT-guided coaxial cutting needle lung biopsies. Chest 2004;126:748-54.

38. O'Neill AC, McCarthy C, Ridge CA, Mitchell P, Hanrahan E, Butler M, et al. Rapid needle-out patient-rollover time after percutaneous CT-guided transthoracic biopsy of lung nodules: effect on pneumothorax rate. Radiology 2012;262:314-9.

39. Kim JI, Park CM, Lee SM, Goo JM. Rapid needle-out patientrollover approach after cone beam CT-guided lung biopsy: effect on pneumothorax rate in 1,191 consecutive patients. Eur Radiol 2015;25:1845-53.

40. Maybody M, Muallem N, Brown KT, Moskowitz CS, Hsu M, Zenobi CL, et al. Autologous blood patch injection versus hydrogel plug in CT-guided lung biopsy: a prospective randomized trial. Radiology 2019;290:547-54.

41. Zaetta JM, Licht MO, Fisher JS, Avelar RL; Bio-Seal Study Group. A lung biopsy tract plug for reduction of postbiopsy pneumothorax and other complications: results of a prospective, multicenter, randomized, controlled clinical study. J Vasc Interv Radiol 2010;21:1235-43.

42. Malone LJ, Stanfill RM, Wang H, Fahey KM, Bertino RE. Effect of intraparenchymal blood patch on rates of pneumothorax and pneumothorax requiring chest tube placement after percutaneous lung biopsy. AJR Am J Roentgenol 2013;200:123843.

43. Babu SB, Srinivasan S, Chung R, Chawla A, Tan HK, Lohan R. Tract sealing with normal saline after percutaneous transthoracic lung biopsies. J Med Imaging Radiat Oncol 2020;64:2114.

44. Khorochkov E, Garvin GJ, Potoczny S, Kozak RI. Injection of saline into the biopsy tract and rapid patient rollover decreases pneumothorax size following computed tomography-guided transthoracic needle biopsy. Can Assoc Radiol J 2018;69:489-92.

45. Choi CM, Um SW, Yoo CG, Kim YW, Han SK, Shim YS, et al. Incidence and risk factors of delayed pneumothorax after transthoracic needle biopsy of the lung. Chest 2004;126:151621.

46. Zhu J, Qu Y, Wang X, Jiang C, Mo J, Xi J, et al. Risk factors associated with pulmonary hemorrhage and hemoptysis follow- 
ing percutaneous CT-guided transthoracic lung core needle biopsy: a retrospective study of 1,090 cases. Quant Imaging Med Surg 2020;10:1008-20.

47. Kim H, Kwon D, Yoon SH, Kim H, Park CM, Goo JM, et al. Bronchovascular injury associated with clinically significant hemoptysis after CT-guided core biopsy of the lung: radiologic and histopathologic analysis. PLoS One 2018;13:e0204064.

48. Kim H, Park CM, Yoon SH, Hwang EJ, Lee JH, Ahn SY, et al. Open bronchus sign on CT: a risk factor for hemoptysis after percutaneous transthoracic biopsy. Korean J Radiol 2018;19:880-7.

49. Ost DE, Ernst A, Lei X, Kovitz KL, Benzaquen S, Diaz-Mendoza J, et al. Diagnostic yield and complications of bronchoscopy for peripheral lung lesions: results of the AQuIRE registry. Am J Respir Crit Care Med 2016;193:68-77. 